\title{
A CHANGE OF RINGS THEOREM
}

LANCE W. SMALL ${ }^{1}$

1. Introduction. Except under special hypotheses it is not possible to relate the global dimension of a ring to that of a factor ring. In this note we shall give such a change of rings theorem which appears to be more general than those in the literature. Applying this theorem, we can determine the global dimension of power series rings over arbitrary Noetherian rings. The theorem is also used to give some estimate of the difference between the left and right global dimensions of certain rings.

2. Notation and definitions. All rings have units and all modules are unitary. $J(R)$ will denote the Jacobson radical of the ring $R$, and $N(R)$ will be the maximal nilpotent ideal, if it should exist, of the ring $R$.

If $M$ is a right (left) $R$-module, we write r.pd $\operatorname{pd}_{R} M\left(1 \cdot \mathrm{pd}_{R} M\right)$ and w.rd ${ }_{R} M\left(\mathrm{w} \mathrm{ld}_{R} M\right)$ for the projective and weak dimensions, respectively. The various global dimensions are written as l.gl.d, r.gl.d and w.gl.d.

\section{Main theorem.}

THEOREM 1. If $R$ is any ring and $I$ is a nilpotent two-sided ideal of $R$, then

$$
\begin{aligned}
& \text { r.gl.d } R \leqq \text { w. } \operatorname{ld}_{R} R / I+\text { r.gl.d } R / I, \\
& \text { l.gl.d } R \leqq \text { w.rd }_{R} R / I+\text { l.gl.d } R / I .
\end{aligned}
$$

If $R$ is right Noetherian and $K$ is any two-sided ideal in $J(R)$, then

$$
\text { r.gl.d } R \leqq \text { w.ld }_{R} R / K+\text { r.gl.d } R / K \text {. }
$$

The proof depends on the following

LEMMA 1. Let $R$ be any ring and I a nilpotent two-sided ideal. If $M$ is a right $R$-module such that $\operatorname{Tor}_{p}^{R}(M, R / I)=0$ (all $\left.p>0\right)$, then $\mathrm{r} \cdot \mathrm{pd}_{R} M=\mathrm{r} \cdot \mathrm{pd}_{R / I} M / M I$.

Proof. We note that if

$$
0 \rightarrow L \rightarrow F \rightarrow M \rightarrow 0
$$

Received by the editors July 18, 1966 and, in revised form, November \&, 1966.

1 This research was partially supported by NSF grant GP 3990. 
is exact with $F$ a free $R$-module, then $\operatorname{Tor}_{p}^{R}(L, R / I)=0$, for all $p>0$, by the connecting homomorphism theorem [4, Chapter V].

First we show that r.pd $\mathrm{pd}_{R} M \geqq \mathrm{r} \cdot \mathrm{pd}_{R / I} M / M I$. Clearly if $M$ is $R$-projective, then $M / M I$ is $R / I$-projective. Assume now that $r . \operatorname{pd}_{R} M=n(0<n<\infty)$ and proceed by induction on $n$. From $\left(^{*}\right)$ r.pd $p_{R} L=n-1$, and since $\operatorname{Tor}_{1}^{R}(M, R / I)=0$ we have the exact sequence of $R / I$-modules (note: $A / A I \approx A \otimes_{R} R / I$, for all right $R$ modules $A$ )

$$
0 \rightarrow L / L I \rightarrow F / F I \rightarrow M / M I \rightarrow 0 .
$$

But, $F / F I$ is $R / I$-free and $\operatorname{Tor}_{p}^{R}(L, R / I)=0(p>0)$. Hence, by induction, r. $\operatorname{pd}_{R / I} L / L I \leqq n-1$ and, thus, r.pd $\operatorname{pd}_{R / I} M / M I \leqq n$ which was to be shown. The assertion is trivial if $\mathrm{r} \cdot \mathrm{pd}_{R} M=\infty$.

The crux of the lemma is to establish r.pd $d_{R} M=\mathrm{r} \cdot \mathrm{pd}_{R / I} M / M I$. Suppose that $M / M I$ is $R / I$-free. Let $\left\{e_{\alpha}\right\}_{\alpha \in A}$ be elements of $M$, the images of which under the natural mapping $M \rightarrow M / M I$ constitute an $R / I$-basis for $M / M I$. Now let $E$ be the submodule of $M$ generated by the $\left\{e_{\alpha}\right\}_{\alpha \in A}$. Since $E+M I=M$ we obtain $(M / E) I$ $=M / E$. But $I$ is nilpotent so that $M / E=0$ and $E=M$. Consider the free $R$-module $F$ with basis $\left\{x_{\alpha}\right\}_{\alpha \in A}$ and $g$ the map such that $g\left(x_{\alpha}\right)=e_{\alpha}$. We now have an exact sequence

$$
0 \rightarrow L \rightarrow F \rightarrow M \rightarrow 0 .
$$

Since $\operatorname{Tor}_{1}^{R}(M, R / I)=0$, we also have the exact sequence (of $R / I$ modules)

$$
0 \rightarrow L \otimes_{R} R / I \rightarrow F \otimes_{R} R / I \stackrel{g \otimes 1}{\longrightarrow} M \otimes_{R} R / I \rightarrow 0 .
$$

But, $g \otimes 1$ is an isomorphism of the free $R / I$-modules $F \otimes_{R} R / I$ and $M \otimes_{R} R / I$. Thus, $L \otimes_{R} R / I=0$ which means $L=L I$ and thus $L=0$. Therefore $M$ is free.

Now assume that $M / M I$ is $R / I$-projective. Consider $\left(^{*}\right)$ again. As before

$$
0 \rightarrow L / L I \rightarrow F / F I \rightarrow M / M I \rightarrow 0
$$

is exact. This last sequence splits: $F / F I \approx L / L I \oplus M / M I$. But, $L / L I \oplus M / M I \approx(L \oplus M) /(L \oplus M) I$ which is free. Hence, $L \oplus M$ is free and $M$ is $R$-projective.

Assume r. $\operatorname{pd}_{R / I} M / M I=n(0<n<\infty)$ and proceed by induction. Just as before $\left({ }^{*}\right)$ gives rise to the exact sequence

$$
0 \rightarrow L / L I \rightarrow F / F I \rightarrow M / M I \rightarrow 0
$$


so that r.pd $\mathrm{pd}_{R / \mathrm{I}} L / L I=n-1$ which, by induction, yields r.pd $\mathrm{pd}_{R} L=n-1$ and, hence, r.pd $\operatorname{pd}_{R} M=n$.

If r.pd $d_{R / I} M / M I=\infty$, then r.pd $\operatorname{pd}_{R} M=\infty$ since r.pd $\operatorname{pd}_{R} M \geqq$ r.pd $\mathrm{pd}_{R / I}$ $M / M I$.

If we examine the proof, we find that the only place we made use of the nilpotence of $I$ was to conclude that if $B I=B$, where $B$ is a right $R$-module, then $B=0$. If $R$ is right Noetherian and our attention is restricted to finitely-generated modules, $B$, then $B I=B$ forces $B=0$ if $I \subset J(R)$ by virtue of the Nakayama lemma. Therefore we obtain

Lemma $1^{\prime}$. Let $R$ be right Noetherian and $I$ a two-sided ideal in $J(R)$. If $M$ is a finitely-generated right $R$-module such that $\operatorname{Tor}_{p}^{R}(M, R / I)=0$ (all $p>0$ ), then r.pd $\operatorname{pd}_{R} M=\mathrm{r} \cdot \mathrm{pd}_{R / I} M / M I$.

It is now possible to prove Theorem 1.

Proof of Theorem 1. It will suffice to establish the first inequality as the others are proved similarly. Let $M$ be an arbitrary right $R$-module and suppose w. $\operatorname{ld}_{R} R / I=n(n<\infty)$. Consider an exact sequence

$$
0 \rightarrow X_{n} \rightarrow F_{n-1} \rightarrow \cdots \rightarrow F_{0} \rightarrow M \rightarrow 0
$$

where the $F_{i}$ are free. By the iterated connecting homomorphism theorem, $\operatorname{Tor}_{p}^{R}\left(X_{n}, R / I\right)=\operatorname{Tor}_{n+p}^{R}(M, R / I) \quad($ all $p>0)$. Since $\mathrm{w} . \mathrm{ld}_{R} R / I=n$, $\operatorname{Tor}_{n+p}^{R}(M, R / I)=0$ (all $p>0$ ). Hence, $X_{n}$ satisfies the condition of Lemma 1 so that r.pd ${ }_{R} X_{n}=\mathrm{r} \cdot \mathrm{pd}_{R / I} X_{n} / X_{n} I \leqq$ r.gl.d $R / I$. To conclude, "paste" a resolution of $X_{n}$ onto

$$
\cdots \rightarrow F_{n-1} \rightarrow \cdots \rightarrow F_{0} \rightarrow M \rightarrow 0 .
$$

Of course, if $R$ is right Noetherian and $M$ is finitely-generated, then $X_{n}, F_{n-1}, \cdots, F_{0}$ may all be taken to be finitely-generated and Lemma $1^{\prime}$ does the trick.

Various special cases of Theorem 1 appear in the literature: see, for example, Kaplansky [3, 1.9].

4. Examples and applications. Auslander-Buchsbaum showed that if $R$ is a commutative Noetherian ring of global dimension $n$ then $R[[x]]$, the ring of formal power series over $R$, has global dimension $n+1$. The next theorem shows that the assumption of commutativity can be dropped.

ThEOREM 2. If $R$ is right Noetherian and r.gl.d $R=n$, then r.gl.d $R[[x]]=n+1$.

Proof. Suppose r.gl.d $R=n<\infty$. Since $x$ is a central nonzero divisor 
in $R[[x]]$, the ideal generated by $x,(x)$, is actually $R[[x]]$-isomorphic

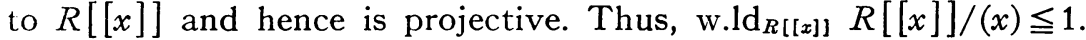
But, $(x) \subset J(R)$ so by Theorem 1 we have r.gl.d $R[[x]] \leqq 1$ + r.gl.d $R[[x]] /(x)=n+1$. On the other hand, r.gl.d $R[[x]] \geqq n+1$ by Theorem 1.3 of Kaplansky [3]. If $n=\infty$, we may use the same device as Auslander-Buchsbaum.

In [5] and [6] the author considered right Noetherian, right hereditary rings. It was found that for such rings $R / N(R)$ was also right hereditary. We use this result now to obtain some information on the left global dimension.

Theorem 3. If $R$ is right Noetherian and r.gl.d $R=1$ and $R / N(R)$ is also left Noetherian, then l.gl.d $R \leqq 2$.

Proof. Since $R / N(R)$ is Noetherian on both sides, we have l.gl.d $R / N(R)=$ r.gl.d $R / N(R) \leqq 1$. We use inequality (2) of Theorem 1 . But, w.rd $R / N(R) \leqq 1$ as $R$ is right hereditary. Thus, 1.gl.d $R \leqq 1$ $+1=2$.

We now turn to an example which will show that the inequalities of Theorems 1 and 3 cannot be strengthened. Let $R$ be the integers localized at a prime and $Q$ be the rationals. Consider the ring $S$ consisting of all $2 \times 2$ matrices

$$
\left(\begin{array}{ll}
r & q_{1} \\
0 & q_{2}
\end{array}\right)
$$

with $r \in R, q_{1}$ and $q_{2} \in Q . S$ is right Noetherian but not left Noetherian, and

$$
J(S)=\left\{\left(\begin{array}{ll}
a & q \\
0 & 0
\end{array}\right) \mid a \in J(R), q \in Q\right\} .
$$

It is not difficult to see that w.ld $J(S)=0$, but 1.pd $J(S)=1$ (see Theorem 2.1 of $[2])$. Since $S / J(S) \approx R / J(R) \oplus Q$, a direct sum of fields, r.gl.d $S / J(S)=0$. Thus, by Theorem 1, r.gl.d $S=1$.

However, l.gl.d $S=2$. For, 1.pd $J(S)=1$ so l.gl.d $S \geqq 2$ and Theorem 3 gives the reverse inequality (since $S / N(S) \approx R \oplus Q$ ). However, if we tried to apply inequality (2) of Theorem 1 to $S$ with $I=J(S)$, we would obtain l.gl.d $S=1$ which is false. Thus, Theorem 1 cannot be generalized in this direction.

Let us now apply inequalities (1) and (2) of Theorem 1 to $S$ with $I=N(S)$. Since $S / N(S) \approx R \oplus Q$ and

$$
\text { w.ld } S / N(S)=\text { w.rd.s } S / N(S)=1 \text {, }
$$

we obtain r.gl.d $S \leqq 2$ and l.gl.d $S \leqq 2$. Hence, in the former case the 
inequality is strict while in the latter it is not. Therefore, the inequalities are "best possible."

AdDED IN Proor. After this paper was written, similar results (in particular, to Lemma $1^{\prime}$ and Theorem 2) appeared in a paper of J. R. Strooker, Lifting projectives, Nagoya Math. J. 27 (1966), 747-749.

\section{BIBLIOGRAPHY}

1. M. Auslander and D. A. Buchsbaum, Homological dimension in Noetherian rings. II, Trans. Amer. Math. Soc. 88 (1958), 194-206.

2. S. U. Chase, A generalization of the ring of triangular matrices, Nagoya Math. J. 18 (1961), 13-25.

3. I. Kaplansky, Homological dimension of rings and modules, Math. Lecture Notes, Univ. of Chicago, 1959.

4. S. Mac Lane, Homology, Springer, Berlin, 1963.

5. L. W. Small, An example in Noetherian rings, Proc. Nat. Acad. Sci. U.S.A. 54 (1965), 1035-1036.

6. - - Hereditary rings, Proc. Nat. Acad. Sci. U.S.A. 55 (1966), 25-27.

U.iversity of California, Berkeley 